\title{
ASPHERICAL GENERATORS OF UNORIENTED COBORDISM
}

\author{
DAVID C. ROYSTER
}

\begin{abstract}
A new set of indecomposable generators for $M O_{*}$ is constructed, each of which is aspherical. Two adjoint maps are used to define these generators. In the process we find a natural basis for $\mathrm{MO}_{*}\left(\mathrm{Z}_{2}\right)$.
\end{abstract}

1. Introduction. Our objective in this paper is to construct a sequence of closed aspherical generators for $M O_{*}$ as a polynomial ring over $\mathrm{Z}_{2}$. Recall that the classical definition of an aspherical manifold from knot theory is a closed connected manifold whose universal covering space is contractible; i.e., these are closed connected $K(\pi, 1)$ manifolds. We will also construct a basis of closed aspherical elements for $M O_{*}\left(\mathbf{Z}_{2}\right)$ as an $M O_{*}$-module in $\$ 3$. However, since the connected sum operation destroys asphericity, it is still unknown if there is a closed connected aspherical representative in each unoriented cobordism class of $M O_{*}$. There are many examples of aspherical manifolds in the literature; cf. [2], [5], [7], [8], and [10]. In the case of flat Riemannian manifolds, all of the known examples bound mod 2. This is true even though there are known examples of closed flat Riemannian manifolds with nonzero Stiefel-Whitney classes; cf. [2] and [7].

In all that follows, $I_{*}\left(\mathbf{Z}_{2}\right)$ will denote the graded unrestricted cobordism ring of smooth manifolds with involution; $M O_{*}\left(\mathrm{Z}_{2}\right)$, the graded unoriented cobordism ring of smooth manifolds with fixed point free involutions; and $M O_{*}$, the graded unoriented Thom cobordism algebra.

2. The maps $\Gamma$ and $\gamma$. Let $S^{1}=\{z \in C|| z \mid=1\}$. Define the map $\Gamma: I_{n}\left(\mathbf{Z}_{2}\right)$ $\rightarrow I_{n+1}\left(\mathbf{Z}_{2}\right)$ as follows: Take $\left\{T, M^{n}\right\}_{2} \in I_{n}\left(\mathbf{Z}_{2}\right)$ and consider the manifold $S^{1} \times M^{n}$ with two involutions $T_{1}(z, m)=(-z, T(m))$ and $T_{2}(z, m)=(\bar{z}, m)$ for $z \in S^{1}$ and $m \in M^{n}$. Observe that $T_{1}$ is fixed point free, so the quotient of $S^{1} \times M^{n}$ by $T_{1}$ is a manifold. $T_{1}$ and $T_{2}$ commute, so $T_{2}$ induces an involution, call it still $T_{2}$, on the quotient manifold $\left(S^{1} \times M^{n}\right) / T_{1}$. Put

$$
\Gamma\left(\left\{T, M^{n}\right\}_{2}\right)=\left\{T_{2},\left(S^{1} \times M^{n}\right) / T_{1}\right\}_{2} \in I_{n+1}\left(\mathbf{Z}_{2}\right) .
$$

Similarly, define the map $\gamma: M O_{n}\left(\mathbf{Z}_{2}\right) \rightarrow M O_{n+1}\left(\mathbf{Z}_{2}\right)$ as follows: Take $\left[\tau, X^{n}\right]_{2} \in M O_{n}\left(Z_{2}\right)$ and consider the manifold $S^{1} \times X^{n}$ with the two fixed point free involutions $T_{3}(z, x)=(\bar{z}, \tau(x))$ and $T_{4}(z, x)=(-z, x)$ for $z \in S^{1}$ and $x \in X^{n} . T_{3}$ and $T_{4}$ commute, so $T_{4}$ induces an involution, call it again $T_{4}$, on the quotient manifold $\left(S^{1} \times X^{n}\right) / T_{3}$. This involution is fixed point

Received by the editors December 9, 1976.

AMS (MOS) subject classifications (1970). Primary 57D85; Secondary 57E99. 
free since $T_{3}$ and $T_{4}$ have no coincidence. Put

$$
\gamma\left(\left[\tau, X^{n}\right]_{2}\right)=\left[T_{4},\left(S^{1} \times X^{n}\right) / T_{3}\right]_{2} \in M O_{n+1}\left(\mathbf{Z}_{2}\right) .
$$

Observe that our definition of the map $\Gamma$ differs slightly from the definition in [1]. To resolve this difference let us consider a differentiable involution $\left(T, M^{n}\right)$. Put $\Gamma\left(\left\{T, M^{n}\right\}_{2}\right)=\left\{T^{\prime}, M^{\prime}\right\}_{2}$, where $M^{\prime}=\left(S^{1} \times M^{n}\right) / T_{1}$ and $T^{\prime}$ is the involution induced on $M^{\prime}$ by $T_{2}$. Now, $T_{1}$ on $S^{1} \times M^{n}$ is fixed point free. The fixed point set of $T_{2}$ on $S^{1} \times M^{n}$ is $\{-1,1\} \times M^{n}$. The coincidence of $T_{1}$ and $T_{2}$ is $\{-i, i\} \times F(T)$, where $F(T)$ is the fixed point set of $T$ on $M^{n}$. Thus, the fixed point set of $T^{\prime}$ on $M^{\prime}$ is the disjoint union of $F(T)$ and $M^{n}$. The normal bundle to this fixed point set is $(\nu \oplus R) \cup R$ where $R$ is the trivial real-line bundle and $\nu$ is the normal bundle to $F(T) \subseteq M^{n}$. Note that the second factor of $R$ appears as the normal bundle to $M^{n}$ in $S^{1} \times M^{n}$. This is exactly the same fixed point data as the map $\Gamma$ in [1]. Thus, the two maps agree on the cobordism level.

2.1. Definition. There is a pairing, which is bilinear as an $M O_{*}$-module homomorphism, $\beta: M O_{*}\left(\mathbf{Z}_{2}\right) \times I_{*}\left(\mathbf{Z}_{2}\right) \rightarrow M O_{*}$ defined as follows: Take $\left[\tau, X^{n}\right]_{2} \in M O_{n}\left(\mathbf{Z}_{2}\right)$ and $\left\{T, M^{k}\right\}_{2} \in I_{k}\left(Z_{2}\right)$ and consider the manifold $X^{n} \times$ $M^{k}$. Define a fixed point free involution on $X^{n} \times M^{k}$ by $T_{1}(x, m)=$ $(\tau(x), T(m))$. Put

$$
\beta\left(\left[\tau, X^{n}\right]_{2},\left\{T, M^{k}\right\}_{2}\right)=\left[\left(X^{n} \times M^{k}\right) / T_{1}\right]_{2} \text { in } M O_{n+k} .
$$

We have the following relationship between the maps $\Gamma$ and $\gamma$.

2.2. THEOREM. The maps $\Gamma$ and $\gamma$ are adjoint with respect to $\beta$.

$$
\beta\left(\gamma\left(\left[\tau, X^{n}\right]_{2}\right),\left\{T, M^{k}\right\}_{2}\right)=\beta\left(\left[\tau, X^{n}\right]_{2}, \Gamma\left(\left\{T, M^{k}\right\}_{2}\right)\right) .
$$

Proof. Put

$$
Y_{1}=\beta\left(\gamma\left(\left[\tau, X^{n}\right]_{2}\right),\left\{T, M^{k}\right\}_{2}\right)=\left[\left(M^{k} \times\left(\left(S^{1} \times X^{n}\right) / T_{3}\right)\right) / T^{\prime}\right]_{2}
$$

and put

$$
Y_{2}=\beta\left(\left[\tau, X^{n}\right]_{2}, \Gamma\left(\left\{T, M^{k}\right\}_{2}\right)\right)=\left[\left(X^{n} \times\left(\left(S^{1} \times M^{k}\right) / T_{1}\right)\right) / T^{\prime \prime}\right]_{2}
$$

Denote a point in $Y_{1}$ by $[m,[z, x]]$ and a point in $Y_{2}$ by $[x,[z, m]]$ for $x \in X^{n}, z \in S^{1}$, and $m \in M^{k}$. Define a map $f: Y_{1} \rightarrow Y_{2}$ by $f([m,[z, x]])=$ $[x,[z, m]]$. This map is a diffeomorphism.

3. The natural basis $\{X(k) \mid k \geqslant 0\}$. We want to consider iterations of the $\operatorname{map} \gamma$ on $\left[A, S^{0}\right]_{2} \in M O_{0}\left(Z_{2}\right)$. Put

$$
X(k)=\gamma^{k}\left(\left[A, S^{0}\right]_{2}\right) \in M O_{k}\left(\mathbf{Z}_{2}\right) \text { for all } k \geqslant 0 .
$$

We have the following properties.

3.1. Proposition. $\Delta(X(k+1))=X(k)$ for all $k \geqslant 0$, where $\Delta$ is the Smith homomorphism.

Proof. We will prove the stronger statement that $\Delta \circ \gamma=$ id. Let $\left[\tau, M^{n}\right]_{2}$ 
$\in M O_{n}\left(\mathbf{Z}_{2}\right)$ and set $\gamma\left(\left[\tau, M^{n}\right]_{2}\right)=\left[\tau^{\prime}, M^{\prime}\right]_{2}$. Let $B=\left\{(z, m) \in S^{1} \times\right.$ $\left.M^{n} \mid \operatorname{Re}(z) \geqslant 0\right\}$ and let $W$ be the image of $B$ in $M^{\prime}$. Then $W \cup \tau^{\prime} W=M^{\prime}$ and $W \cap \tau^{\prime} W=\partial W=M^{n}$. By 26.2 of $[3], \Delta\left(\left[\tau^{\prime}, M^{\prime}\right]_{2}\right)=\left[\tau^{\prime}, \partial W\right]_{2}=$ $\left[\tau, M^{n}\right]_{2}$.

3.2. Proposition. $\varepsilon(X(k))=0$ for all $k>0$, where $\varepsilon: M O_{*}\left(\mathbf{Z}_{2}\right) \rightarrow M O_{*}$ is the augmentation map.

Proof. We will again prove a stronger statement: $\varepsilon \circ \gamma=0$. Let $\left[\tau, M^{n}\right]_{2}$ $\in M O_{n}\left(\mathbf{Z}_{2}\right) . M^{n}$ may be regarded as the total space of a line bundle, $\eta$, over $M^{n} / \tau$. By applying $\gamma$ to $\left[\tau, M^{n}\right]_{2}$, we add a trivial line bundle to $\eta$. Then by applying $\varepsilon$, we pass to the projective bundle $R P(\eta \oplus R)$. Thus,

$$
\varepsilon\left(\gamma\left(\left[\tau, M^{n}\right]_{2}\right)\right)=[R P(\eta \oplus R)]_{2} \in M O_{n+1} .
$$

Now, $\eta \oplus R$ is a two plane bundle over $M^{n} / \tau$ with no second StiefelWhitney class. By 2.1 of $[4],[R P(\eta \oplus R)]_{2}=0$.

3.3. Proposition. $\{X(k) \mid k \geqslant 0\}$ forms a homogeneous $\mathrm{MO}_{*}$-module basis for $\mathrm{MO}_{*}\left(\mathrm{Z}_{2}\right)$.

Proof. It is sufficient to show that $\mu(X(k))$ is a generator of $H_{k}\left(R P(\infty) ; \mathbf{Z}_{2}\right)$ for each $k \geqslant 0$, where $\mu: M O_{*}\left(\mathbf{Z}_{2}\right) \rightarrow H_{*}\left(R P(\infty) ; \mathbf{Z}_{2}\right)$ is the Thom homomorphism. Consider the following commutative diagram

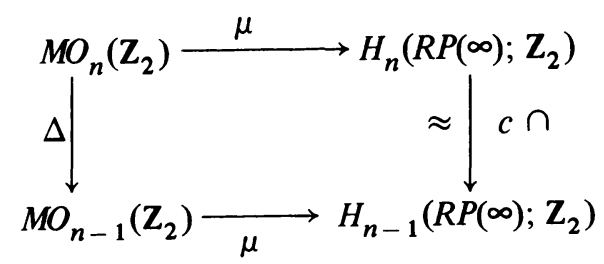

where $c$ is the generator of $H^{1}\left(R P(\infty) ; \mathbf{Z}_{2}\right)$. Our proof will proceed by induction. Clearly, $\mu(X(0))$ is a generator of $H_{0}\left(R P(\infty) ; \mathbf{Z}_{2}\right)$. Assume that $\mu(X(k))$ is a generator of $H_{k}\left(R P(\infty) ; \mathbf{Z}_{2}\right)$ for all $k<n$. For $n$, we have that

$$
c \cap \mu(X(n))=\mu(\Delta(X(n)))=\mu(X(n-1)) \text {, }
$$

which is a generator of $H_{n-1}\left(R P(\infty) ; \mathbf{Z}_{2}\right)$. Since capping with $c$ is an isomorphism, $\mu(X(n))$ is a generator of $H_{n}\left(R P(\infty) ; \mathbf{Z}_{2}\right)$.

These three propositions give us the main theorem of this section.

3.4. THEOREM. The basis $\{X(k) \mid k \geqslant 0\}$ is unique with respect to satisfying (3.1)-(3.3).

Proof. Let $\left\{Y_{k} \mid k \geqslant 0\right\}$ be another homogeneous basis for $M_{*}\left(Z_{2}\right)$ such that (i) $\Delta\left(Y_{k+1}\right)=Y_{k}$ for all $k \geqslant 0$, and (ii) $\varepsilon\left(Y_{k}\right)=0$ for all $k>0$. It suffices to show that $Y_{k}+X(k)=0$ for all $k \geqslant 0$. Clearly, $Y_{0}=X(0)$. Assume that $Y_{k}+X(k)=0$ for all $k<n$, and suppose that $Y_{n}+X(n) \neq 0$. Since $\{X(k) \mid k \geqslant 0\}$ is a homogeneous basis, we have a unique representation 


$$
Y_{n}+X(n)=\sum_{j=0}^{n} a_{j} X(j), \quad a_{j} \in M O_{n-j}
$$

Now,

$$
\begin{aligned}
0 & =Y_{n-1}+X(n-1)=\Delta\left(Y_{n}+X(n)\right) \\
& =\Delta\left(\sum_{j=0}^{n} a_{j} X(j)\right)=\sum_{j=1}^{n} a_{j} X(j-1) .
\end{aligned}
$$

Thus, $a_{j}=0$ for $1 \leqslant j \leqslant n$ and $Y_{n}+X(n)=a_{0} X(0), a_{0} \in M O_{n}$. Also, $0=$ $\varepsilon\left(Y_{n}+X(n)\right)=a_{0} \varepsilon(X(0))$. But $\varepsilon(X(0))=1 \in M O_{0}$. Thus, $a_{0}=0$ and we have our contradiction.

With this basis, we have a much more simple representation of any element in $M O_{*}\left(\mathrm{Z}_{2}\right)$.

3.5. Proposition. For any $\left[\tau, M^{n}\right]_{2} \in M O_{n}\left(\mathrm{Z}_{2}\right)$,

$$
\left[\tau, M^{n}\right]_{2}=\sum_{j=0}^{n} \varepsilon\left(\Delta^{j}\left(\left[\tau, M^{n}\right]_{2}\right)\right) X(j)
$$

Proof. This follows from repeated applications of $\Delta$ and $\varepsilon$. We have that

$$
\left[\tau, M^{n}\right]_{2}=\sum_{j=0}^{n} a_{j} X(j), \quad a_{j} \in M O_{n-j}
$$

Now,

$$
\Delta^{j}\left(\left[\tau, M^{n}\right]_{2}\right)=a_{j} X(0)+\sum_{i=j+1}^{n} a_{i} X(i-j) \text { for } 0 \leqslant j \leqslant n .
$$

Then, $\varepsilon\left(\Delta^{j}\left(\left[\tau, M^{n}\right]_{2}\right)\right)=a_{j}$.

3.7. Corollary. $\left[A, S^{n}\right]_{2}=\sum_{j=0}^{n}[R P(n-j)]_{2} X(j)$.

3.8. Note. Since $X(k)=\gamma^{k}\left(\left[A, S^{0}\right]_{2}\right)=\gamma^{k-1}\left(\left[A, S^{1}\right]_{2}\right)$, it is clear that $X(k)$ is a closed aspherical manifold for all $k \geqslant 0$.

4. Aspherical indecomposable generators. Let lis consider $R P(2)$ with the involution $T$ defined in homogeneous coordinates by $T\left[x_{1}, x_{2}, x_{3}\right]=$ $\left[-x_{1}, x_{2}, x_{3}\right]$. The fixed point set of $T$ is the disjoint union of a point and $R P(1)$.

4.1. THEOREM. $\varepsilon\left(\Gamma^{k}\left(\{T, R P(2)\}_{2}\right)\right)$ cobords for $k$ odd and is indecomposable for $k$ even.

Proof. Recall that $[M]_{2}$ is indecomposable in $M O_{*}$ if it cannot be expressed as a sum of products of lower dimensional classes.

If $k$ is odd, then 4.4 of [4] shows that $\varepsilon\left(\Gamma^{k}\left(\{T, R P(2)\}_{2}\right)\right)$ cobords. Now, if $k$ is even, let $\nu$ denote the normal bundle to the fixed point set of $T$ on $R P(2)$. By 4.2 of [4], $\varepsilon\left(\Gamma^{k}\left(\{T, R P(2)\}_{2}\right)\right)$ is indecomposable if and only if $[R P(\nu \oplus(k$ $+1) R)]_{2}$ is indecomposable. The restriction of $\nu$ to $R P(1)$ is the canonical twisted real-line bundle, $\xi \rightarrow R P(1)$. Therefore, 


$$
[R P(\nu \oplus(k+1) R)]_{2}=[R P(k+2)]_{2}+[R P(\xi \oplus(k+1) R)]_{2}
$$

By 2.2 of $[4],[R P(\xi \oplus(k+1) R)]_{2}=0$. Since $k$ is even, $[R P(k+2)]_{2}$ is known to be indecomposable.

Of course, this theorem only depends on the unrestricted cobordism class of $(T, R P(2))$. Let us consider the 2-torus on which there is an involution with 4 isolated fixed points. Remove an open disk about either one or three of these fixed points. Note that the involution is fixed point free on the boundaries of these disks. Replace these disks with closed Moebius bands by identifying along the boundaries. We want the following involution on each of the Moebius bands. We may consider the Moebius band as the one-cell bundle over $R P(1)$. The involution on the Moebius band will be induced by the antipodal map on the boundary curve. Clearly, the fixed point set of this involution on the Moebius band is $R P(1)$. Let us call the manifold obtained from the 2-torus in this way $W^{2}$, and the involution induced on $W^{2}, T^{\prime}$. Then $W^{2}$ is aspherical and $\left\{T^{\prime}, W^{2}\right\}_{2}=\{T, R P(2)\}_{2}$. We have the following immediate corollary.

4.2. Corollary. $\varepsilon\left(\Gamma^{k}\left(\left\{T^{\prime}, W^{2}\right\}_{2}\right)\right)$ cobords for $k$ odd and is indecomposable for $k$ even. Furthermore, each of these manifolds is aspherical.

Proof. The first part is clear from 4.1, and the second part follows from the homotopy exact sequence for a covering space and the fact that $W^{2}$ is aspherical.

So, we have found closed aspherical indecomposable generators for $M O_{n}$ for $n$ even. However, for $n$ odd we need some further results.

4.3. Definition. Define a map $g: M O_{*} \rightarrow I_{*}\left(\mathrm{Z}_{2}\right)$ by $g\left(\left[M^{n}\right]_{2}\right)=\left\{S, M^{n} \times\right.$ $\left.M^{n}\right\}_{2}$, where $S$ is the switching involution $S(x, y)=(y, x)$.

4.4. Lemma. The map $g$ is a well-defined, additive, and multiplicative homomorphism.

Proof. To show that $g$ is well defined, it suffices to show that if $\left[M^{n}\right]_{2}=0$, then $g\left(\left[M^{n}\right]_{2}\right)=0$. To compute $g\left(\left[M^{n}\right]_{2}\right)=\left\{S, M^{n} \times M^{n}\right\}_{2}$, we must look at the normal bundle to $F(S)$, the fixed point set of $S$. Now, $F(S) \cong M^{n}$. Let $\nu \rightarrow F(S)$ denote the normal bundle to $F(S)$. Now, $\nu$ is equivalent to the tangent bundle of $M^{n}, \tau\left(M^{n}\right)$. Thus, it is sufficient to show that all of the characteristic numbers of $\tau\left(M^{n}\right)$ vanish. By the assumption that $\left[M^{n}\right]_{2}=0$, all of the characteristic numbers of $\tau\left(M^{n}\right)$ are zero.

To show that $g$ is additive, we must show that $\{S,(M \cup N) \times(M \cup N)\}_{2}$ $=\{S, M \times M\}_{2}+\{S, N \times N\}_{2}$. Thus, it suffices to show that $\{S, N \times M$ $\cup M \times N\}_{2}=0$. Since $N \times M$ and $M \times N$ are diffeomorphic, it suffices to show that $\{S,(M \times N) \cup(M \times N)\}_{2}=0$. Consider the manifold $M \times N$ $\times I$ with the involution $T$ defined by $T(m, n, t)=(m, n, 1-t)$. Now, $\partial(M$ $\times N \times I)=(M \times N) \cup(M \times N)$ and $T \mid \partial(M \times N \times I)=S$. Therefore, $\{S,(M \times N) \cup(N \times M)\}_{2}=0$.

The fact that $g$ is multiplicative is easy to show. 
Let us define decomposable and indecomposable elements in $I_{*}\left(Z_{2}\right)$ as in $M O_{*}$. Note then that $g$ takes decomposable elements to decomposable elements since $g$ is additive and multiplicative. We need the following two lemmas before proceeding.

4.5. LemMA. Let $\left\{T, M^{n}\right\}_{2} \in I_{*}\left(Z_{2}\right)$; then $\beta\left(\left[A, S^{0}\right]_{2},\left\{T, M^{n}\right\}_{2}\right)=\left[M^{n}\right]_{2}$ $\in M O_{*}$.

The proof of this lemma is really quite simple and will be left to the reader.

4.6. LEMMA. If $\{T, M\}_{2} \in I_{*}\left(Z_{2}\right)$ is decomposable, then $\beta\left(X(j),\{T, M\}_{2}\right)$ is decomposable.

Proof. Since $\beta$ is bilinear, we may assume that $\{T, M\}_{2}=\{\varphi, V\}_{2}$. $\{\psi, W\}_{2}$. Then

$$
\begin{aligned}
Y & =\beta\left(X(j),\{\varphi, V\}_{2} \cdot\{\psi, W\}_{2}\right)=\beta\left(\gamma^{j}\left(\left[A, S^{0}\right]_{2}\right),\{\varphi, V\}_{2} \cdot\{\psi, W\}_{2}\right) \\
& =\beta\left(\left[A, S^{0}\right]_{2}, \Gamma^{j}\left(\{\varphi, V\}_{2} \cdot\{\psi, W\}_{2}\right)\right) \\
& =\beta\left(\left[A, S^{0}\right]_{2}, \Gamma^{j-1}\left(\Gamma\left(\{\varphi, V\}_{2}\right) \cdot\{\psi, W\}_{2}+\varepsilon\left(\{\varphi, V\}_{2}\right) \cdot \Gamma\left(\{\psi, W\}_{2}\right)\right)\right)
\end{aligned}
$$

by Theorem 1.3 of [1]. So,

$$
\begin{aligned}
Y= & \beta\left(\left[A, S^{0}\right]_{2}, \Gamma^{j-1}\left(\Gamma\left(\{\varphi, V\}_{2}\right) \cdot\{\psi, W\}_{2}\right)\right) \\
& +[V]_{2} \beta\left(\left[A, S^{0}\right]_{2}, \Gamma^{j}\left(\{\psi, W\}_{2}\right)\right)
\end{aligned}
$$

from the fact that $\beta$ is a bilinear, $M O$-map, and from the properties of $\Gamma$ in [1]. By descent, (4.7) yields

$$
\begin{aligned}
Y & =\beta\left(\left[A, S^{0}\right]_{2}, \Gamma^{j}\left(\{\varphi, V\}_{2}\right) \cdot\{\psi, W\}_{2}\right)+\text { decomposables } \\
& =\left[\Gamma^{j}\left(\{\varphi, V\}_{2}\right)\right]_{2} \cdot[W]_{2}+\text { decomposables }
\end{aligned}
$$

by (4.5).

Let $n \in \mathbf{Z}^{+}$be odd. There are $r, s \in \mathbf{Z}^{+}$such that $n+1=2^{r}(2 s+1)$. Let $j=2^{r}-1$ and $k=s 2^{r}$. Dold has shown [6] that if $n$ is not of the form $2^{i}-1$, then

$$
[P(j, k)]_{2}=\left[S^{j} \times C P(k) /(x, z) \sim(-x, \bar{z})\right]_{2}
$$

is an indecomposable generator of $M O_{n}$. From this definition of $P(j, k)$, it is clear that

$$
[P(j, k)]_{2}=\beta\left(\left[A, S^{j}\right]_{2},\{C, C P(k)\}_{2}\right),
$$

where $C$ is complex conjugation on $C P(k)$. From 3.7 we have that $\left[A, S^{j}\right]_{2}=$ $\sum_{i=0}^{j}[R P(j-i)]_{2} X(i)$. So, we now have

$$
[P(j, k)]_{2}=\sum_{i=0}^{j}[R P(j-i)]_{2} \beta\left(X(i),\{C, C P(k)\}_{2}\right) \text {. }
$$

It is well known [3], [9] that $\{C, C P(k)\}_{2}=\{S, R P(k) \times R P(k)\}_{2}=$ $g\left([R P(k)]_{2}\right)$. Now, $k$ is always even, so $[R P(k)]_{2}$ is indecomposable in $M O_{k}$. 
Recall the involution $\left(T^{\prime}, W^{2}\right)$ from above, and set $\left[V_{k}\right]_{2}=$ $\varepsilon\left(\Gamma^{k-2}\left(\left\{T^{\prime}, W^{2}\right\}_{2}\right)\right)$. Now, $\left[V_{k}\right]_{2}$ is indecomposable in $M O_{k}$ by 4.2. Thus, $[R P(k)]_{2}$ and $\left[V_{k}\right]_{2}$ can differ only by decomposables. We have that $[R P(k)]_{2}$ $=\left[V_{k}\right]_{2}+\left[Y_{k}\right]_{2}$, where $\left[Y_{k}\right]_{2}$ is a sum of decomposable elements. So, we now have that $\{C, C P(k)\}_{2}=g\left(\left[V_{k}\right]_{2}+\left[Y_{k}\right]_{2}\right)$. (4.8) now yields that

So,

$$
[P(j, k)]_{2}=\sum_{i=0}^{j}[R P(j-i)]_{2} \beta\left(X(i), g\left(\left[V_{k}\right]_{2}+\left[Y_{k}\right]_{2}\right)\right) \text {. }
$$

$$
\begin{aligned}
{[P(j, k)]_{2}=} & \beta\left(X(j), g\left(\left[V_{k}\right]_{2}\right)\right)+\beta\left(X(j), g\left(\left[Y_{k}\right]_{2}\right)\right) \\
& +\sum_{i=0}^{j-1}[R P(j-i)]_{2} \beta\left(X(i), g\left(\left[V_{k}\right]_{2}+\left[Y_{k}\right]_{2}\right)\right) .
\end{aligned}
$$

Clearly, $[R P(j-i)]_{2} \beta\left(X(i), g\left(\left[V_{k}\right]_{2}+\left[Y_{k}\right]_{2}\right)\right)$ is decomposable in $M O_{*}$ for $0<i \leqslant j-1$. By 4.6, $\beta\left(X(j), g\left(\left[Y_{k}\right]_{2}\right)\right)$ is also decomposable. Therefore, since $[P(j, k)]_{2}$ is indecomposable, $\beta\left(X(j), g\left(\left[V_{k}\right]_{2}\right)\right)$ must also be indecomposable.

From our definition of $\beta$, we know that $X(j) \times V_{k} \times V_{k}$ is the total space of a covering space for $\beta\left(X(j), g\left(\left[V_{k}\right]_{2}\right)\right)$. Since both $X(j)$ and $V_{k}$ are closed aspherical manifolds, $\beta\left(X(j), g\left(\left[V_{k}\right]_{2}\right)\right)$ is also a closed aspherical manifold. Thus, $[P(j, k)]_{2}$ in $M O_{n}, n$ odd, is equivalent to a closed connected aspherical indecomposable element modulo decomposable elements. Let $D_{*}$ denote the graded ideal of decomposable elements in $M O_{*}$. We have proven the following.

4.10. TheOREM. For each odd $n \in \mathbf{Z}^{+}$there is a closed connected aspherical manifold $M(n)$ such that $[M(n)]_{2}$ is indecomposable and $[M(n)]_{2}=[P(j, k)]_{2}$ $\bmod D_{*}$.

The author would like to thank P. E. Conner for suggesting this problem and for his many helpful conversations.

\section{REFERENCES}

1. J. C. Alexander, The bordism ring of manifolds with involution, Proc. Amer. Math. Soc. 31 (1972), 536-542.

2. L. Auslander and R. H. Szczarba, Characteristic classes of compact solvmanifolds, Ann. of Math. (2) 76 (1962), 1-8.

3. P. E. Conner and E. E. Floyd, Differentiable periodic maps, Ergebnisse Grenzgebiete, no. 33, Springer-Verlag, Berlin and New York, 1964.

4. __ Fibering within a cobordism class, Michigan Math. J. 12 (1965), 33-47.

5. P. E. Conner and F. Raymond, Actions of compact Lie groups on aspherical manifolds, Proc. Conf. on Manifolds (Univ. of Georgia, Athens, Ga., 1969), pp. 227-264.

6. A. Dold, Erzeugende der Thomschen Algebra $\Re$, Math. Z. 65 (1956), 25-35.

7. R. Lee and R. H. Szczarba, On the integral Pontrjagin classes of a Riemannian flat manifold, Geometrie Dedicata 3 (1974), 1-10.

8. A. T. Vasquez, Flat Riemannian manifolds, J. Differential Geometry 4 (1970), 367-382.

9. C. T. C. Wall, Determination of the cobordism ring, Ann. of Math. (2) 72 (1960), 292-311.

10. The topological space-form problem, Proc. Conf. on Manifolds (Univ. of Georgia, Athens, Ga., 1969), pp. 319-331.

Department of Mathematics, Louisiana State University, Baton Rouge, louisiana 70803 